\title{
SELF-ORGANIZATION OF COVALENTLY-BONDED rGO AND PbS QDS FOR OPTOELECTRONIC DEVICES
}

\author{
Aleksandr LITVIN, Anton BABAEV, Peter PARFENOV \\ ITMO University, St. Petersburg, Russian Federation, \\ litvin@itmo.ru
}

https://doi.org/10.37904/nanocon.2019.8523

\begin{abstract}
Lead sulfide ( $\mathrm{PbS}$ ) quantum dots (QDs) have been covalently attached to reduced graphene oxide ( $\mathrm{rGO}$ ). Thin films from a hybrid rGO-PbS nanomaterial have been formed by a Langmuir-Blodgett technique. We found that oleic acid on QDs surface induces self-organization of high-quality rGO-PbS films with a thickness of 1-2 monolayer. To utilize these films in optoelectronic devices, post-deposition ligand exchange procedures have been applied. We found both high conductivity and light-sensitivity in EDT- and TBAI-treated rGO-PbS films. We have applied rGO-PbS hybrid films to enhance the performance of a hybrid and heterojunction QD-based solar cells.
\end{abstract}

Keywords: Quantum dots, graphene, PbS, optoelectronics, self-organization

\section{INTRODUCTION}

Hybrid nanomaterials based on light-harvesting quantum dots (QDs) and highly conductive 2D nanosheets attract much attention for creation new optoelectronic devices [1]. Thanks to the quantum confinement effect, QDs allow precise tuning of an absorption band position by varying a QD size [2]. Depending on the semiconductor material of the QDs, the absorption spectrum can cover a wide spectral range from UV to NIR $[3,4]$. Near-infrared QDs (PbS, PbSe, CIS, AIS) attract enormous attention due to their possible application in solar energy harvesting, night vision and telecommunication systems [5]. The energy absorbed by a QD can be nonradiatively transferred to a $2 \mathrm{D}$ material by resonant energy transfer or charge transfer [6]. Possessing high carrier mobilities, 2D materials (such as graphene and transition metal dichalcogenides) provide good transport of extracted charge carriers between electrodes $[7,8]$.

For the successful utilization of such hybrid nanomaterials in optoelectronic devices, universal methods of creating nanostructures based on them with nanoscale control of their parameters are required [9]. Covalent bonding of QDs and graphene-related materials proved to be a good method for creation a hybrid material with desired and controllable distance between QDs and graphene. Short bonding molecules provide close distance between nanomaterials ensuring effective charge and energy transfer processes.

In the current study we demonstrate that covalently bonded reduced graphene oxide ( $\mathrm{rGO}$ ) and PbS QDs can form large-scale monolayered films. Being tightly attached to $\mathrm{GGO}$ by a silane linker, PbS QDs remains covered by long oleic acid ligands, which induce a formation of self-organized film at the water-air interface. Such a film can be simultaneously transferred to several substrates, and its properties can be tuned by a post-deposition ligand-exchange, keeping its uniformity. We demonstrate that they possess properties suitable for their utilization in optoelectronic devices.

\section{METHODS}

PbS QDs with diameter of $4.0 \mathrm{~nm}$ were synthesized by a hot-injection method [10]. rGO and 95\% (3Mercaptopropyl) trimethoxysilane (MPTS) were purchased from Sigma-Aldrich. The hybrid nanomaterial 
based on covalently bonded rGO and PbS QDs was prepared by slightly modified method proposed by MartínGarcía et al [11]. MPTS linker is used to bond QDs to rGO surface as shown in a Figure 1.
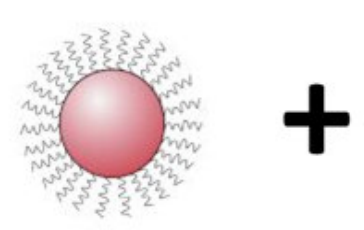

PbS QD<smiles>CO[Si](C)(CCCS)OC</smiles>

MPTS

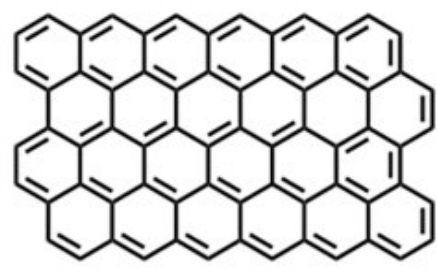

rGO

Figure 1 The hybrid nanomaterial consists of PbS QDs and rGO bonded by a silane linker

MPTS-functionalized rGO and PbS QDs were mixed in a solution first. The mixture has been shaken for 2 hours at $1500 \mathrm{rpm}$ to provide better hybrid material formation, then the mixture was diluted with toluene in 1:2 proportions and centrifuged at $9000 \mathrm{rpm}$. The supernatant with unattached QDs was removed and the precipitate was dissolves in chloroform with ultrasonication. The obtained solution was used for LangmuirBlodgett (LB) films preparation.

\section{RESULTS AND DISCUSSION}

First, we have paid additional attention to the precipitation procedure. Employing the Raman and time-resolved photoluminescence (PL) spectroscopy, we analyzed different paths to remove unreacted products. We have found that conventional washing with $\mathrm{EtOH}$ and centrifugation is less effective than washing with toluene. PL lifetime quenching indicates a formation of PbS-rGO hybrid, while longer PL lifetimes indicate unreacted QDs. The measured PL decay times are listed in a Table 1. PL decay time obtained for MPTS-treated PbS QDs was used as a reference. Using both toluene and $\mathrm{EtOH}$ for precipitation leads to separation of attached and unreacted QDs. However, according to obtained PL decay times, toluene allows to wash more unattached QDs and does not result in significant damage of the rGO-PbS hybrid. After the additional precipitation with $\mathrm{EtOH}$ afterwards, supernatant does not bring better efficiency. Because after an additional precipitation both supernatant and precipitate shown similar PL lifetimes, we can conclude that a second washing step lead exclusively to a separation of rGO-PbS complexes into two parts. Thus, we believe that a one-step toluene precipitation should be used for preparation of the hybrid inks. A formation of hybrid PbS-rGO complexes in a precipitate was confirmed by quenched PL decay from PbS QDs and Raman spectra from rGO. Raman spectra obtained for pure rGO and different precipitates are shown in Figure 2. After the washing and redispersion, few drops of a hybrid inks were placed onto an air/liquid interface. After compression, a dense film was formed. This LB film can be transferred onto a substrate at the dipping rate of $1 \mathrm{~mm} / \mathrm{min}$ speed.

Table 1 Calculated PL decay times after different precipitation

\begin{tabular}{|c|c|c|}
\hline Solvent used & PL decay time percipitate (ns) & PL decay time supernatant (ns) \\
\hline Toluele & 145 & 395 \\
\hline $\mathrm{EtOH}$ & 170 & 340 \\
\hline Toluene+EtOH & 125 & 140 \\
\hline Reference & \multicolumn{2}{|c|}{440} \\
\hline
\end{tabular}




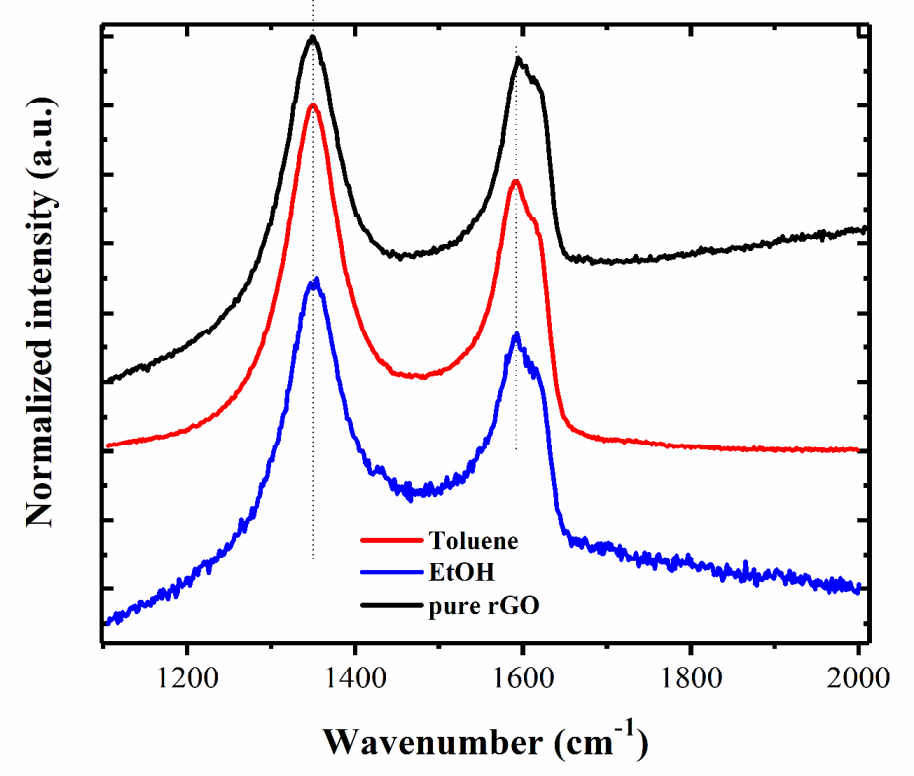

Figure 2 Raman spectra obtained was a pure rGO and precipitates obtained after toluene and EtOH washing

The used LB approach allows fabrication a high-quality hybrid films with a thickness of $10-20 \mathrm{~nm}$ that corresponds to 1 and 2 layers of $\mathrm{rGO}$ with $\mathrm{PbS}$ QDs attached at both sides. Figure 3 shows scanning electron microscopy images of PbS-rGO films transferred on a patterned ITO glass. As can be seen from the left panel, large scale and uniform film can be fabricated. To cover a pixelated ITO substrate for a device fabrication, a direction of dipping should be kept in mind. A right panel displays two pixels with a high quality rGO-PbS film (upper pixel) and an area where formation of the film started (lower panel).
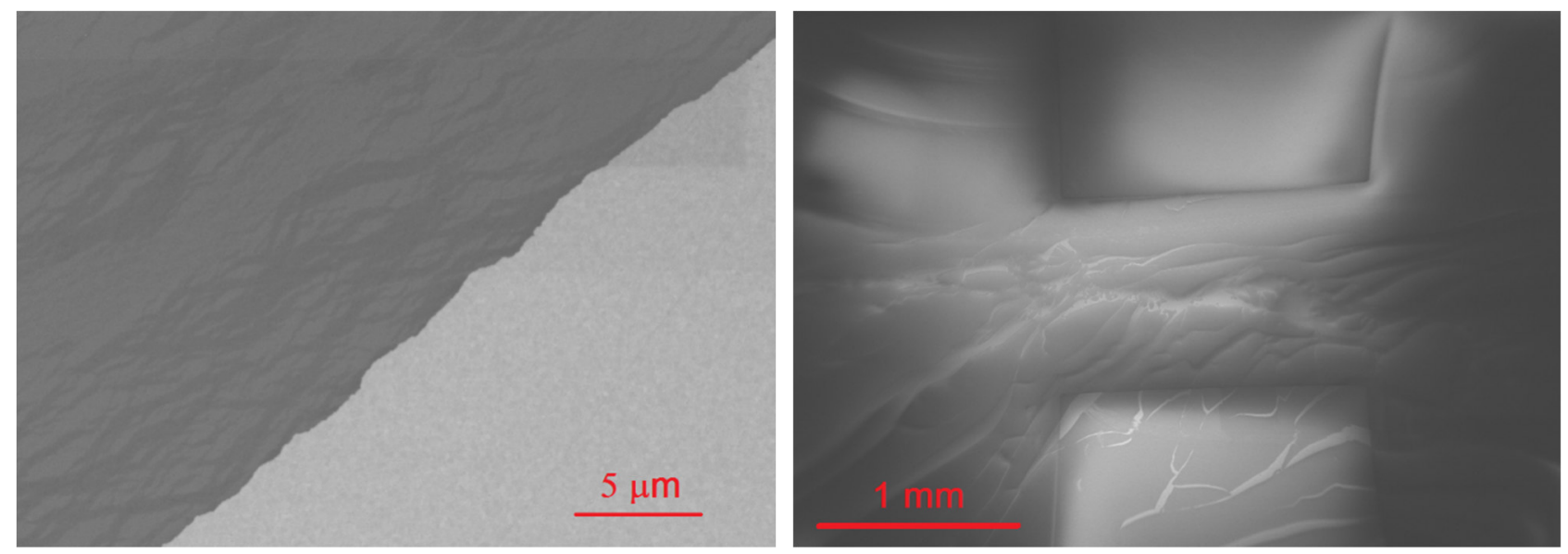

Figure 3 SEM images of the hybrid LB filmed from rGO and PbS QDs on a pixelated ITO substrate

After deposition the film, native oleic acid ligands can be removed by post-deposition ligand-exchange procedures. Post-deposition ligand-exchange allows fabrication more compact QD film with better conductivity. Since the native ligands are long fatty molecules, a hopping transport of charge carriers cannot be realized. After post-deposition ligand-exchange QDs are covered by shorter molecules or even atoms, that allows effective hopping of a charge carriers between QDs. To improve electronic properties of rGO-PbS QD films, we performed post-deposition ligand-exchange with EDT (1,2-Ethandithiol) and TBAI (Tetrabutylammonium iodide) solutions. 
To do the conductivity measurements, a $1.5 \mathrm{~mm}$ groove was etched in ITO layer on the ITO covered glass substrate, as shown in a Figure 4 (right panel). The groove and ITO sides were covered by a rGO-PBs film, and relation between current through and voltage across the film in the groove was measured. We used a four-wire connection by Keithley 2636B SourceMeter instead of the standard 4-probe method was not enough sensitive. The conductivity was calculated using cross-section area $(11 \mathrm{~mm} \times 20 \mathrm{~nm})$ and the width of the groove $(1.5 \mathrm{~mm})$ at $20 \mathrm{~V}$ voltage. We observed that conductivity increased dramatically after the post-deposition ligand-exchange procedures. The largest increase was in the EDT sample and smaller in the TBAI-exchanged sample. But the conductivity of the first one reduced in twelve times for a day, while for the second one the reducing was much smaller (40\%). It says that stability of TBAI films is much better. The data are listed in a Table 2.

Table 2 The results of electrical measurements for LB rGO-PbS QDs film before and after post-deposition ligand-exchange

\begin{tabular}{|c|c|c|c|c|c|}
\hline Sample & Day & Current (pA) & $\begin{array}{c}\text { Resistance, G } \Omega \\
(@ 20 \mathrm{~V})\end{array}$ & $\begin{array}{c}\text { Resistitivity, } \\
\Omega \times m\end{array}$ & $\begin{array}{c}\text { Conductivity, } \\
\text { Sm/cm }\end{array}$ \\
\hline PbS rGO LB & 0 & 47 & 426 & $63000^{*}$ & $1.6 \times 10^{-7 *}$ \\
\hline PbS-TBAI rGO LB & 0 & 806 & 25 & 3670 & $2.7 \times 10^{-6}$ \\
\hline PbS-EDT rGO LB & 0 & 1243 & 16 & 2347 & $4.3 \times 10^{-6}$ \\
\hline PbS rGO LB & 1 & 54 & 370 & $45000^{*}$ & $2.2 \times 10^{-7 *}$ \\
\hline PbS-TBAI rGO LB & 1 & 500 & 40 & 5880 & $1.7 \times 10^{-6}$ \\
\hline PbS-EDT rGO LB & 1 & 98 & 204 & 30000 & $3.3 \times 10^{-7}$ \\
\hline
\end{tabular}
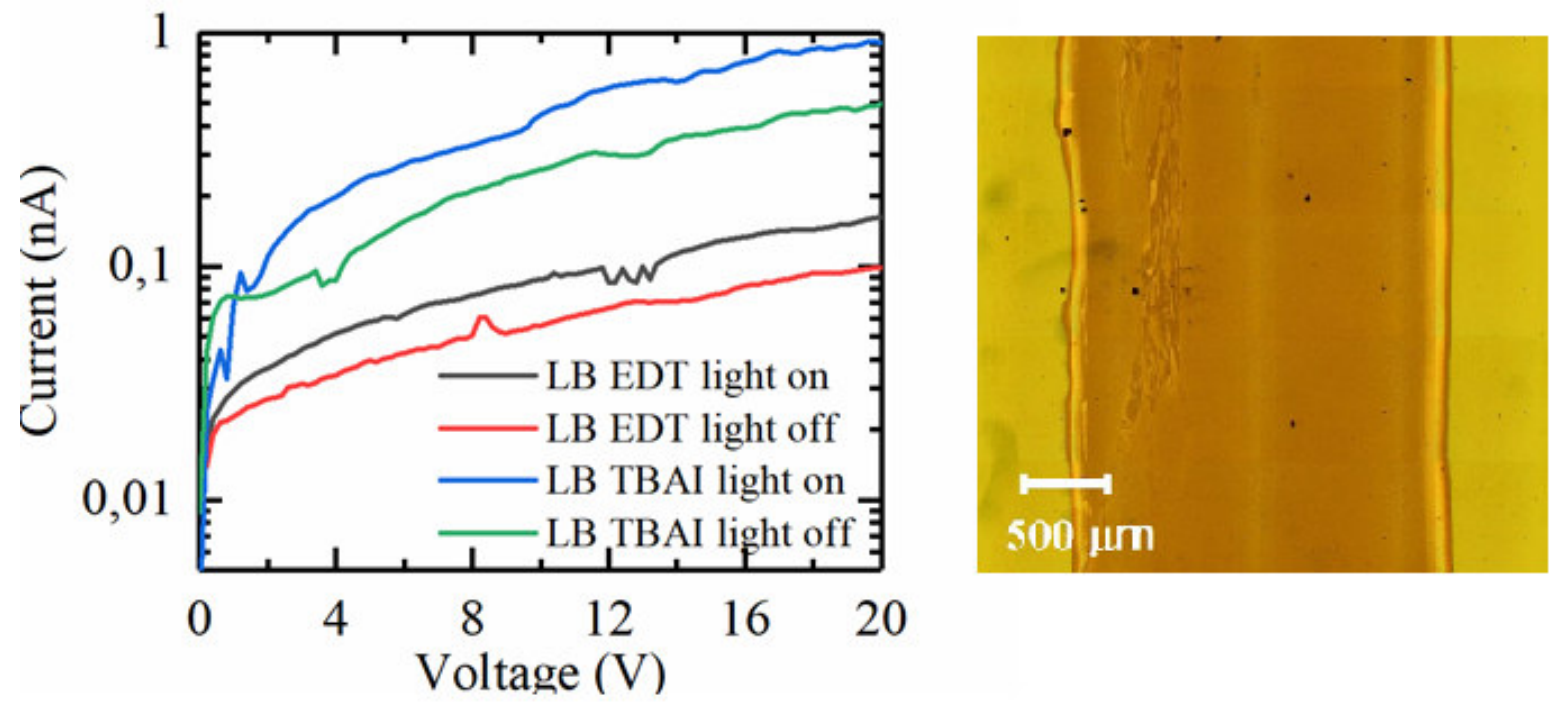

Figure 4 IV-curves for rGO-PbS QDs LB films with and without illumination (left). The ITO glass with an etched strip was used to estimate rGO-PbS QDs LB films conductivity (right).

Importantly, the films demonstrate light-sensitive electrical response. Under illumination the films by halogen lamp (intensity $1.5 \mathrm{~W} / \mathrm{m}^{2}$, so exposition was $0.77 \mu \mathrm{W}$ on sample), we observed an increase of current for both TBAI- and EDT-treated films as shown in a Figure 4 (left panel). The observed increase of conductivity was about $60 \%$ for the both samples. That indicates that PbS QDs absorb a radiation and transfer their carriers to $\mathrm{rGO}$, which provides better conductivity between electrodes. 


\section{CONCLUSION}

To conclude, we demonstrated an optimized method for creation of hybrid films composed of PbS QDs and rGO with a monolayer thickness control. We showed that such films demonstrate excellent optoelectronic properties after the post-deposition ligand-exchange procedures. Strong interaction between QDs and rGO allows increasing current density in the film under light illumination. We believe that the method may be extended for other $0 \mathrm{D}-2 \mathrm{D}$ systems for creation of efficient phototransistors and photodiodes.

\section{ACKNOWLEDGEMENTS}

The authors thank the Ministry of Education and Science of the Russian Federation Grant no. 14.584.21.0032 (ID RFMEFI58417X0032). A.P.L. thanks the Ministry of Education of the Russian Federation for financial support (Scholarship of the President of the Russian Federation for young scientists and graduate students, SP-70.2018.1)

\section{REFERENCES}

[1] WU, Jianghong, LU, Yanghua, FENG, Sirui, WU, Zhiqian, LIN, Shuyuan, HAO, Zhenzhen, YAO, Tianyi, Li, Xinming, Zhu, Hongwei and LIN, Shisheng. The interaction between quantum dots and graphene: the applications in graphene-based solar cells and photodetectors. Adv. Funct. Mater. 2018, vol 28, no. 50, p. 1804712.

[2] PIETRYGA, Jeffrey M., PARK, Young-Shin, LIM, Jaehoon, FIDLER, Andrew F., BAE, Wan K., BROVELI, Sergio and LIMOV, Victor I. Spectroscopic and Device Aspects of Nanocrystal Quantum Dots. Chem. Rev. 2016, vol. 116, pp. 10513 - 10622.

[3] XU, Gaixia, ZENG, Shuwen, ZHANG, Butian, SWIHAT, Mark T. YONG, Ken-Tye and PRASAD, Paras N. New generation cadmium-free quantum dots for biophotonics and nanomedicine. Chem. Rev. 2016, vol. 116, pp. 12234 - 12327.

[4] LITVIN, Aleksandr, PARFENOV, Peter, USHAKOVA, Elena, FEDOROV, Anatoly, ARTEMYEV, Mikhail, PRUDNIKAU, Anatoly, CHEREVKOV, Sergey, RUKHLENKO, Ivan, and BARANOV, Alexander. Size-dependent room-temperature luminescence decay from PbS quantum dots. Proc. of SPIE. 2012, p. 85641Z-85641Z-7.

[5] SHRESTHA, Aabhash, BATMUNKH, Munkhbayar, TRICOLI, Antonio, DAI, Sheng and QIAO, Shi Z. Recent advance in near-infrared active lead chalcogenide quantum dots: preparation, post-synthesis ligand exchange and applications in solar cells. Angew. Chem. 2019, vol. 58, no. 16, pp. 5202-5224.

[6] GUZELTURK, Burak and DEMIR, Hilmi V. Near-field energy transfer using nanoemitters for optoelectronics. Adv. Funct. Mater. 2016, vol. 26, no. 45, pp. 8158-8177.

[7] LIU, Zhike, LAU, Shu P. and YAN, Feng. Functionalized graphene and other two-dimensional materials for photovoltaic devices: device design and processing. Chemical Society Reviews. 2015, vol. 44, no,15, pp. 56385679 .

[8] YIN, Zongyou, ZHU, Jixin, HE, Qiyuan, CAO, Xiehong, TAN, Chaoliang, CHEN, Hongyu, YAN, Qingyu and ZHANG, Hua. Graphene-Based Materials for Solar Cell Applications. Adv. Energy Mater. 2014, vol. 4, no. 1, p. 1300574.

[9] ZHUGE, Fuwei, ZHENG, Zhi, LUO, Peng, LV, Liang, HUANG, Yu, LI, Huiqiao and ZHAI, Tianyou. Nanostructured materials and architectures for advanced infrared photodetection. Adv. Mater. Technol. 2017, vol 2, no. 8, p. 1700005.

[10] USHAKOVA, Elene, LITVIN, Aleksandr, PARFENOV, Peter, FEDOROV, Anatoly, ARTEMYEV, Mikhail, PRUDNIKAU, Anatoly, RUKHLENKO, Ivan and BARANOV, Alexander. Anomalous size-dependent decay of lowenergy luminescence from PbS quantum dots in colloidal solution. ACS Nano. 2012, vol. 6, no. 10, pp. 89138921.

[11] MARTÍN-GARCÍA, Beatriz, POLOVITSYN, Anatolii, PRATO, Mirko and MOREELS, Ivan. Efficient charge transfer in solution-processed PbS Quantum Dot-reduced graphene oxide hybrid materials. J. Mater. Chem. C. 2015, Vol. 3 , no. 27 , pp. $7088-7095$. 